\section{Tolerance to Fusarium Root Rot and Changes in Antioxidative Ability in Mycorrhizal Asparagus Plants}

\author{
Abu Shamim Mohammad Nahiyan \\ The United Graduate School of Agricultural Science, Gifu University, 1-1 \\ Yanagido, Gifu 501-1193, Japan
}

\author{
Yoh-ichi Matsubara ${ }^{1}$ \\ Faculty of Applied Biological Sciences, Gifu University, 1-1 Yanagido, Gifu \\ 501-1193, Japan
}

Additional index words. arbuscular mycorrhizal fungi, asparagus decline, Fusarium oxysporum f. sp. asparagi, growth promotion, symbiosis

Abstract. Tolerance to fusarium root rot caused by Fusarium oxysporum f. sp. asparagi (Foa, MAFF305556 and N9-31) and the changes in antioxidative abilities in mycorrhizal asparagus (Asparagus officinalis L., cv. Welcome) plants were investigated. Asparagus plants were inoculated with arbuscular mycorrhizal fungus (AMF, Glomus sp. R10) and Foa was inoculated 10 weeks after AMF inoculation. AMF plants accumulated higher dry weight of ferns and roots than non-AMF plants before and after Foa inoculation. AMF colonization level reached more than $70 \%$ and no difference noted among the treatments. As for disease tolerance, non-AMF plants showed $100 \%$ in incidence of root rot and highest severity in both Foa isolates; the severity of symptom was relatively higher in MAFF305556 compared with N9-31. However, AMF plants showed lower severity than non-AMF plants in both Foa isolates. Before and after Foa inoculation, antioxidative abilities increased in most of the AMF plants than non-AMF in the following items: activity of superoxide dismutase (SOD) and ascorbate peroxidase (APX), 1,1-diphenyl-2-picrylhydrazyl (DPPH) radical scavenging activity, and total contents of polyphenol and ascorbic acids. These results suggest that plant growth enhancement and tolerance to fusarium root rot appeared in mycorrhizal asparagus plants. In this case, the disease tolerance might be associated with the increase in antioxidative ability.

Asparagus (Asparagus officinalis L.) is a low-input, high-market value and long-term perennial vegetable crop with a production cycle of up to 15 years or more (Hamel et al., 2005; Yergeau et al., 2006). It is a rich source of phytochemicals such as flavonoids (e.g., rutin and anthocyanins), other phenolic and polyphenolic compounds, saponins, etc., which have biological and medicinal impact on human health (Hartung et al., 1990). However, asparagus decline is a serious and increasing threat in asparagus-producing regions over the world (Hamel et al., 2005; Knaflewski et al., 2008; Reid et al., 2002; Wong and Jeffries, 2006). Asparagus decline is typified by a decrease in yields with a reduction in spear size and number and then eventual death of plants within a few years of planting (Wong and Jeffries, 2006). As a result, asparagus is eventually abandoned and alternative crops are planted. The problem is exacerbated by a replant phenomenon such that additional loss is incurred if fields are

Received for publication 27 Dec. 2011. Accepted for publication 6 Feb. 2012.

This study was supported by Grants-in-Aid for Scientific Research (No. 21580029), Japan Society for the Promotion of Science (JSPS).

${ }^{1}$ To whom reprint requests should be addressed; e-mailymatsu@gifu-u.ac.jp.
$500 \mathrm{~W}$ ) plants (Matsubara et al., 2003); however, many points remain unclear about the mechanisms of disease tolerance in mycorrhizal plants. In pathogen stress conditions, production of a higher concentration of reactive oxygen species (ROS) such as $\mathrm{H}_{2} \mathrm{O}_{2}$, superoxide anion $\left(\mathrm{O}_{2}^{-}\right)$, and hydroxyl radical has been shown to create cytotoxic conditions (Sahoo et al., 2007). To overcome this negative consequence of ROS, plants have evolved various protective mechanisms either to reduce or completely eliminate antioxidative abilities of producing antioxidative enzymes and substances under environmental stresses such as plant disease, drought, and temperature (Moghaddam et al., 2006; Sahoo et al., 2007). As for mycorrhizal plants, Garmendia et al. (2006) reported that disease tolerance and an increase in SOD activity occurred in pepper, and drought tolerance and antixodative enzymes (activity of SOD and APX) increased in mycorrhizal citrus plants (Wu et al., 2006). In addition, Zhu et al. (2010) demonstrated that tolerance to high-temperature stress and an increase in antioxidative enzymes occurred in mycorrhizal maize plants. However, it has been unclear how antioxidative factors change through symbiosis with AMF in asparagus plants and how the changes are associated with disease tolerance.

In this study, the influence of AMF colonization on tolerance to fusarium root rot and the changes in antioxidative ability in mycorrhizal asparagus plants were investigated to clarify the mechanisms of disease tolerance.

\section{Materials and Methods}

Inoculation of arbuscular mycorrhizal fungus. Seeds of asparagus (Asparagus officinalis L., cv. Welcome) were sown in commercial soil (autoclaved at $1.2 \mathrm{~kg} \cdot \mathrm{cm}^{-2}$ and $121^{\circ} \mathrm{C}$ for $1 \mathrm{~h})$ in a plastic container $(43 \times 27 \times 17 \mathrm{~cm})$. During the time of seed sowing, plant holes were made; each hole contained $3 \mathrm{~g} /$ plant commercial AMF (Glomus sp. R10) inocula supplied by Idemitsukosan Co. Ltd. (Tokyo, Japan). Then, seeds were sown onto the inocula, finally covered with soil, and administered with mixed fertilizer (13N:11P:13K, $0.5 \mathrm{~g}$ per plant). Forty plants per plot with three replications were irrigated as regularly and grown in a greenhouse.

Inoculation of Fusarium oxysporum $f$. $s p$. asparagi. Two strains of Foa (MAFF305556 and N9-31) were grown on potato dextrose agar media. The conidia were harvested in potato sucrose liquid media and incubated at $25{ }^{\circ} \mathrm{C}$ in the dark for $7 \mathrm{~d}$. The conidial suspension was sieved and the concentrations adjusted to $10^{6}$ conidia $/ \mathrm{mL}$. Ten weeks after AMF inoculation, each plant was inoculated by pouring $50 \mathrm{~mL}$ of the conidial suspension onto the soil.

Evaluation of arbuscular mycorrhizal fungus colonization level. Ten weeks after AMF inoculation and 8 weeks after Foa inoculation, roots of asparagus were preserved with $70 \%$ ethanol and stained according to Phillips and Hayman (1970). The rate of AMF colonization in 1-cm segments of lateral roots 
(abbreviated RFCSL) was calculated. Hence, RFCSL expresses the percentage of $1-\mathrm{cm}$ AMF-colonized segments to the total $1-\mathrm{cm}$ segments of all lateral roots; the number of total segments was $\approx 30$ per plant. Average colonization was calculated from the values of five plants in each time.

Estimation of symptoms of fusarium root rot. Eight weeks after Foa inoculation, the symptoms (reddish brown root lesion and transparent rotted part of roots) of fusarium root rot were rated to 6 degrees as follows: 0 , no symptom; frequency of diseased storage roots in a root system: 1 , less than $20 \% ; 2$, $20 \%$ to $40 \% ; 3,40 \%$ to $60 \% ; 4,60 \%$ to $80 \%$; and $5,80 \%$ to $100 \%$. In addition, the disease index was calculated by the following formula:

$$
\begin{aligned}
& \text { Disease index }= \\
& \sum \text { (number of plants } \\
& \quad \times \text { number of degree in symptoms) } \\
& \quad \text { Total number of plants } \\
& \quad \times 5 \text { (maximum degree in symptom) }
\end{aligned}
$$

Analysis of antioxidative abilities. Ten weeks after AMF inoculation and 8 weeks

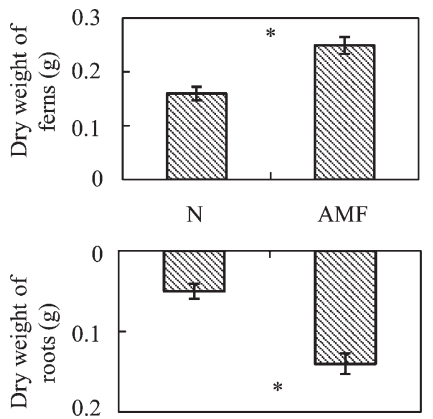

Fig. 1. Dry weight of ferns and roots in mycorrhizal asparagus plants 10 weeks after arbuscular mycorrhizal fungus (AMF) inoculation. $\mathrm{N}=$ nonAMF-inoculated plants; $\mathrm{AMF}=$ Glomus sp. R10. Bars represent SES $(\mathrm{n}=10)$. *Significant difference between non-AMF and AMF plants ( $t$ test, $P \leq 0.05)$ after Foa inoculation, 10 plants were sampled and partitioned into ferns and roots and frozen in liquid nitrogen. Analysis of antioxidative enzyme activities and antioxidative substances were carried out according to the methods of Beauchamp and Fridovich (1971) (SOD), Wu et al. (2006) (APX), Burits and Bucar (2000) (DPPH radical scavenging activity), Folin and Denis (1915) (polyphenol), and Roe et al. (1948) (ascorbic acid), respectively.

Statistical analysis. Mean values were separated by $t$ test for dry weight (10 weeks after AMF inoculation), RFCSL, and antioxidative abilities at $P \leq 0.05$. Dry weight (8 weeks after Foa inoculation) was analyzed by Tukey's multiple range test at $P \leq 0.05$. All analyses were performed using XLSTAT pro statistical analysis software (Addinsoft, New York, NY).

\section{Results}

Ten weeks after AMF inoculation, AMF plants significantly enhanced the dry weight of ferns and roots compared with the nonAMF plants (Fig. 1). AMF colonization occurred successfully and reached up to $63 \%$, 10 weeks after AMF inoculation (data not shown). As for antioxidative ability, 10 weeks after AMF inoculation, SOD and APX activity were higher in AMF plots than non-AMF in both ferns and roots (Fig. 2). On the other hand, DPPH radical scavenging activity and polyphenol contents increased in most of the plant parts of AMF plots than non-AMF, except ferns in polyphenol contents. Ascorbic acid contents showed no difference in both ferns and roots between AMF and non-AMF plots.

Eight weeks after Foa inoculation, nonAMF plants showed $100 \%$ in incidence of root rot and highest severity in both Foa isolates; the severity of symptom was relatively higher in MAFF305556 compared with N9-31(Fig. 3); no disease symptom appeared in the plants without Foa inoculation (data not shown). However, AMF plants showed lower severity than non-AMF plants, and the severity of symptoms varied depending on Foa isolates; MAFF305556 plants showed relatively lower incidence and severity than N9-31 in AMF plants. The disease indices of fusarium root rot reached more than 80 in non-AMF plants of the Foa isolates, whereas it was low as 37 in MAFF 305556 and 57 in N9-31 of AMF plants (Fig. 4). Hence, the disease indices and incidence of fusarium root rot for the AMF and non-AMF plants followed a similar pattern. As for growth condition of asparagus plants 8 weeks after Foa inoculation, dry weight of ferns and roots was greater in AMF plants than non-AMF in both Foa (Fig. 5). AMF colonization level (RFCSL) reached more than $70 \%$ and no differences were noted between the treatments (Fig. 6).

In the analysis of antioxidative ability 8 weeks after Foa inoculation, AMF plants showed higher SOD activity in both ferns and roots than non-AMF plants (Fig. 7). APX activity was higher in ferns of AMF plants, but no difference appeared in roots of AMF and non-AMF. On the other hand, DPPH radical scavenging activity increased in roots of AMF plants, whereas polyphenol and ascorbic acid contents were higher in both ferns and roots of AMF plants compared with nonAMF.

\section{Discussion}

In this study, dry weight of ferns and roots increased in all the AMF plants compared with non-AMF before and after Foa inoculation, which indicates the growth enhancement through symbiosis appeared in mycorrhizal asparagus plants. Previous reports revealed similar results indicating that AMF had a growth-promoting effect in host plants (Ozgonen and Erkilic, 2007; Wu et al., 2006). As for tolerance to fusarium root rot in this experiment, both incidence and severity of symptoms were reduced by AMF, suggesting that tolerance to fusarium root rot appeared in mycorrhizal asparagus plants. Matsubara et al. (2003) reported that AMF increased root rot tolerance in asparagus (cv. Mary Washington $500 \mathrm{~W}$ ) plants; our results in 'Welcome' agreed
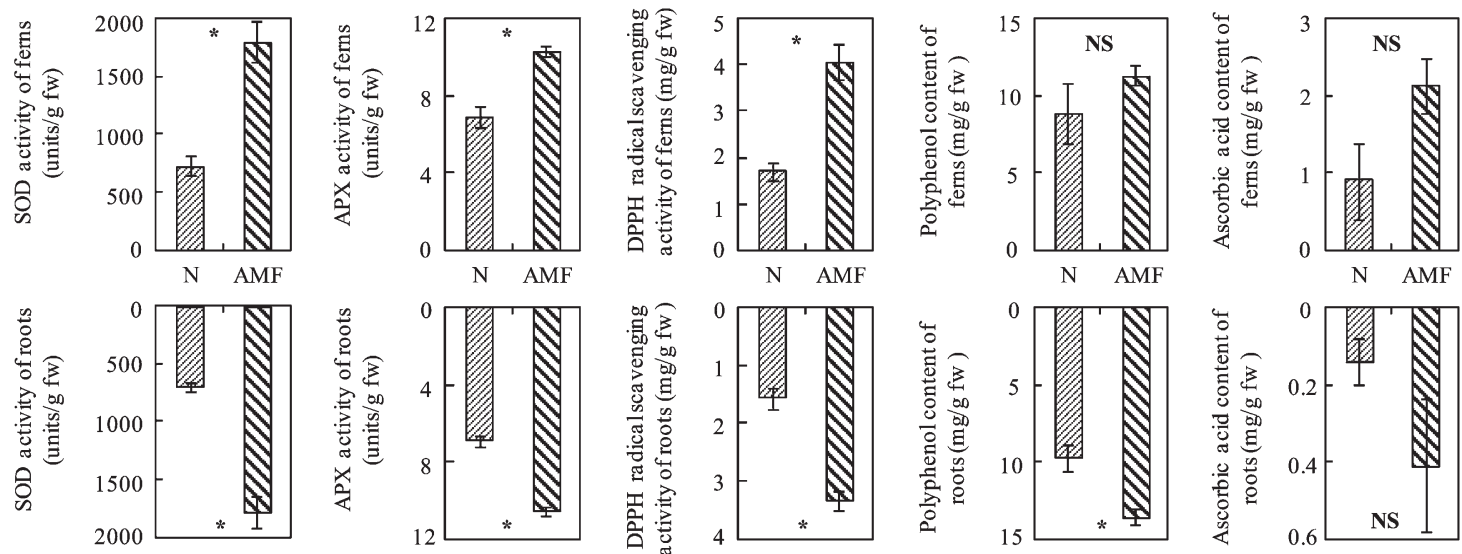

Fig. 2. Superoxide dismutase (SOD), ascorbate peroxidase (APX), 1,1-diphenyl-2-picrylhydrazyl (DPPH) radical scavenging activity, polyphenol, and ascorbic acid contents in ferns and roots 10 weeks after arbuscular mycorrhizal fungus (AMF) inoculation. N = non-AMF-inoculated plants; AMF $=$ Glomus sp. R10. Bars represent SE $(\mathrm{n}=10)$. *Significant difference between non-AMF and AMF plants $(t$ test, $P \leq 0.05)$; NS $=$ nonsignificant. 


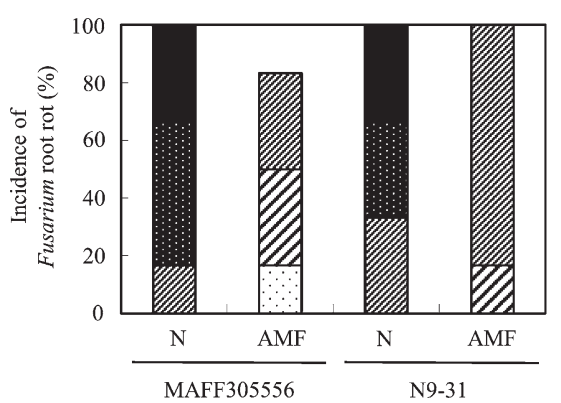

Fig. 3. Incidence of fusarium root rot in asparagus plants 8 weeks after Fusarium oxysporum f. sp. asparagi (Foa) inoculation. Ratio of diseased roots in a root system: $(\square),-20$;

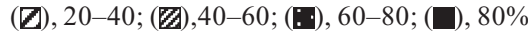
to $100 \%$. N = non-AMF-inoculated; $\mathrm{AMF}=$ Glomus sp. R10; MAFF305556 and N9-31, Foa.

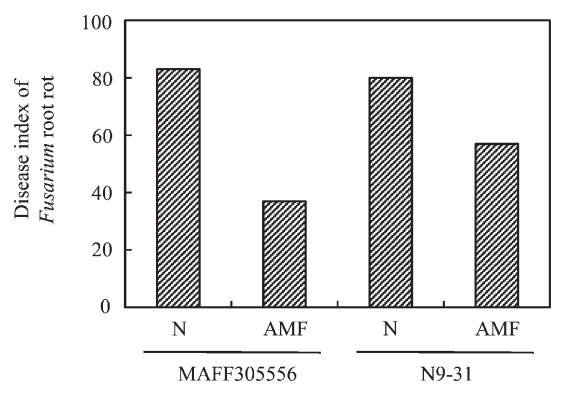

Fig. 4. Disease indices of fusarium root rot in asparagus plants 8 weeks after Fusarium oxysporum f. sp. asparagi (Foa) inoculation. $\mathrm{N}=$ non-AMF-inoculated; AMF = Glomus sp. R10; MAFF305556 and N9-31, Foa.

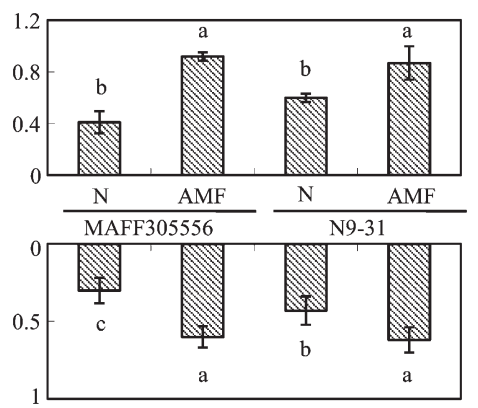

Fig. 5. Dry weight of ferns and roots in mycorrhizal asparagus plants 8 weeks after Fusarium oxysporum f. sp. asparagi (Foa) inoculation. $\mathrm{N}=$ non-AMF-inoculated plants; $\mathrm{AMF}=$ Glomus sp. R10; MAFF305556 and N9-31, Foa. Bars represent SES $(n=10)$. Columns denoted by different letters indicate significant difference by Tukey's test $(P=$ $0.05)$.

with those findings. Norman et al. (1996) reported that the incidence of the symptom caused by Phytophthora fragariae in strawberry plants was reduced by the inoculation of AMF, although the effect differed with AMF species. Ozgonen and Erkilic (2007) reported

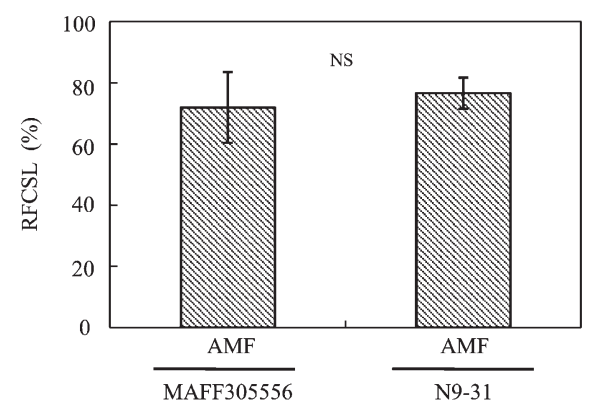

Fig. 6. Arbuscular mycorrhizal fungus (AMF) colonization level (RFCSL) in asparagus plants 8 weeks after Fusarium oxysporum f. sp. asparagi (Foa) inoculation. AMF = Glomus sp. R10 MAFF305556 and N9-31, Foa. Bars represent SES $(\mathrm{n}=5)$. NS $=$ indicates no significant difference between the treatments ( $t$ test, $P \leq 0.05$ ).

that growth promotion and tolerance to Phytophthora capsici had no correlation with the mycorrhizal colonization levels in peppers. In the present experiment, we could not clarify AMF fungal difference in disease tolerance, and no characteristic relationship between colonization levels and disease tolerance appeared. On the other hand, Sutton (1973) demonstrated that AMF colonization consisted of three phases: 1) a leg phase during which spore germination, germ tube growth, and initial penetration occur; 2) a rapid growth phase, coinciding with the development of external mycelium, and spread of the fungus within the roots; and 3) a stable phase during which the proportion of infected roots to non-infected ones remains nearly constant. In this study, colonization level was checked only twice so that it is difficult to estimate when AMF reached the maximum colonization level during the experimental period and how the colonization level affects the disease tolerance.

Some reports described that AMF colonization itself induced a temporary increase in antioxidative abilities such as SOD, guaiacol peroxidase, catalase, APX, and flavonoid content, suggesting that colonization might be temporary stress for host plants. (Blilou et al., 2000; Salzer et al., 1999; Volpin et al., 1995; Wu et al., 2006; Zhu et al., 2010). In this study, SOD, APX, and DPPH radical scavenging activity in both ferns and roots and polyphenol contents in roots increased in all the AMF plants before Foa inoculation. In the aspects of such antioxidative factors, our results support their findings. However, ascorbic acid contents did not increase before Foa inoculation, so it is difficult to estimate completely whether AMF colonization is a stress factor for asparagus plants.

SOD acts as a defensive reaction and detoxifies $\mathrm{O}_{2}^{-}$among the antioxidative enzymes; thus, SOD activity is considered the most important key enzyme in antioxidative abilities in plants (Fridovich, 1986). Sahoo et al. (2007) mentioned that SOD activity increased in Phytopthora blight in taro under induced blight condition compared with controls. In mycorrhizal pepper plants, increase in SOD activity and disease tolerance appeared in pathogen (Verticillium dahliae) stress conditions (Garmendia et al., 2006). Moghaddam et al. (2006) mentioned that SOD activity was higher in a resistant strawberry cultivar than susceptible cultivars with Mycosphaerella fragariae infection. In the present study, tolerance to fusarium root rot appeared in mycorrhizal asparagus plants, and SOD and APX activity increased in most of the parts of AMF plants after Foa inoculation. Our results showed similar patterns to those findings because the increase in SOD activity related to disease tolerance. From these findings, antioxidative enzyme activity might be closely related with disease tolerance in mycorrhizal plants. However, in this study, analysis of antioxidative abilities was carried out only twice; on the contrary, it is difficult to clarify the detailed relationship between disease tolerance and antioxidative enzyme abilities. Further studies would be needed in this context to increase the frequency of analysis of antioxidative abilities both before and after Foa inoculation with a short interval.

Antioxidative substances such as polyphenol contents have lower electron reduction potential than the electron reduction potential of oxygen radicals; as a result, polyphenol contents directly scavenge reactive oxygen intermediates without promoting further oxidative reactions (Ainsworth and Gillespie, 2007). Vanitha et al. (2009) mentioned that total phenol content increased in bacterial wilt in tomato on pathogen inoculation. Hichem et al. (2009) reported that salt stress induced DPPH free radical scavenging activity and polyphenolic compounds in maize. In mycorrhizal St. John's wort plants, increased ascorbic acid content and disease tolerance appeared in pathogen (Colletotrichum gloeosporioides) stress conditions (Richter et al., 2011). From these facts, antioxidative substances have some relation with stress factors. Our results showed similar patterns to those findings as the increase in DPPH radical scavenging activity, polyphenol, and ascorbic acid contents in most of the Foa-inoculated AMF plots. Hence, antioxidative substances also have an association with disease tolerance in mycorrhizal asparagus plants, the same as antioxidative enzymes.

On the other hand, Pozo et al. (2002) reported that in tomato plants with a split root system, tolerance to Phytophthora parasitica appeared in both non-AMFinoculated roots and inoculated roots in AMF plants, so that induced systemic disease tolerance was recognized. In this study, some of the antioxdative abilities increased in ferns, where no colonization occurred. From these facts, we estimate the induced systemic disease tolerance in mycorrhizal asparagus plants with a split root system for fusarium disease in addition to fern disease such as stem blight and the relationship between antioxidative ability and induced disease tolerance.

In conclusion, these results suggest that tolerance to fusarium root rot was induced in 

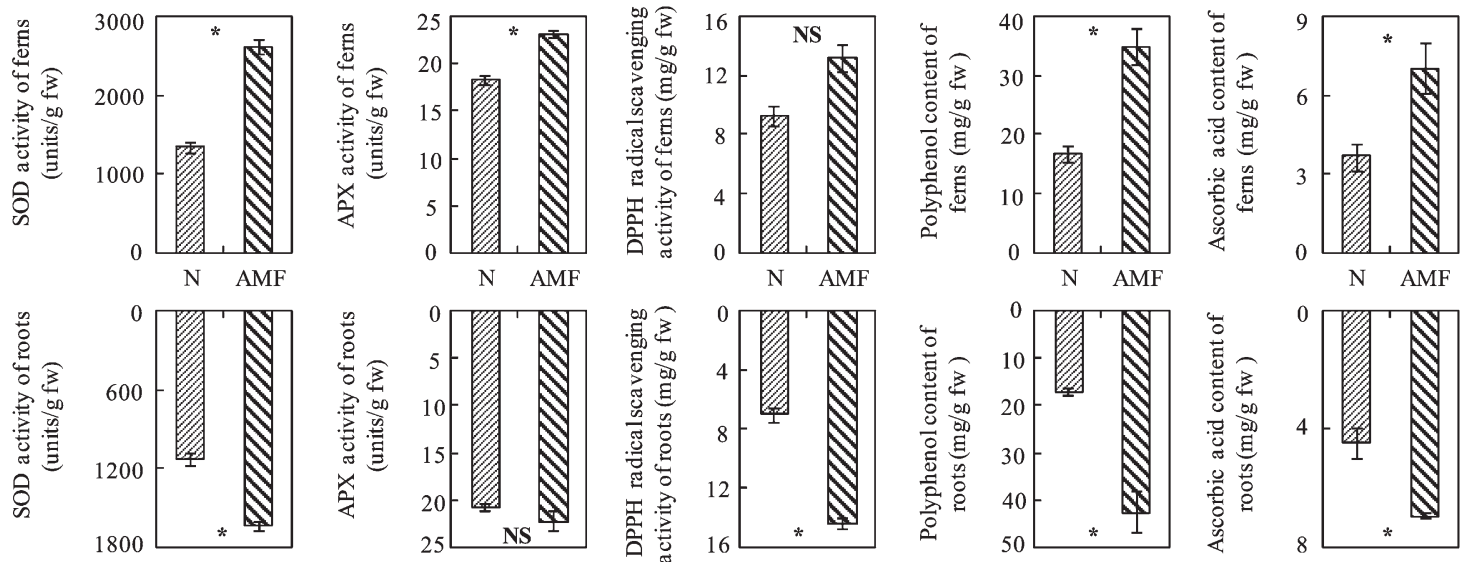

Fig. 7. Superoxide dismutase (SOD), ascorbate peroxidase (APX), 1,1-diphenyl-2-picrylhydrazyl (DPPH) radical scavenging activity, polyphenol, and ascorbic acid contents in ferns and roots of mycorrhizal asparagus plants 8 weeks after Fusarium oxysporum f. sp. asparagi (Foa) (MAFF 305556) inoculation. N= nonAMF-inoculated plants; AMF = Glomus sp. R10. Bars represent SES $(\mathrm{n}=10) . *$ Significant difference between non-AMF and AMF plants $(t$ test, $P \leq 0.05)$; NS $=$ nonsignificant.

asparagus plants by AMF, and disease tolerance has an association with the changes in antioxidative abilities. This proposal seeks to develop a sustainable practice to manage the disease and improve plant health, thus contributing to an improvement in asparagus decline.

\section{Literature Cited}

Ainsworth, E.A. and K.M. Gillespie. 2007. Estimation of total phenolic content and other oxidation substrates in plant tissues using Folin-Ciocalteu reagent. Nat. Protoc. 2:875-877.

Beauchamp, C. and I. Fridovich. 1971. Superoxide dismutase: Improved assays and an assay applicable to acrylamide gels. Anal. Biochem. 44:276-287.

Blilou, I., P. Bueno, J.A. Ocampo, and J.M. Garrido. 2000. Induction of catalase and ascorbate peroxidase activities tobacco roots inoculated with the arbuscular mycorrhizal Glomus mosseae. Mycol. Res. 104:722-725.

Blok, W.J. and G.J. Bollen. 1995. Fungi on roots and stem bases of asparagus in the Netherlands: Species and pathogenicity. Eur. J. Plant Pathol. 101:15-24.

Blok, W.J., M.J. Zwankhuizen, and G.J. Bollen. 1997. Biological control of Fusarium oxysporum f. sp. asparagi by applying non-pathogenic isolates of F. oxysporum. Biocont. Sci. Tech. 7:527-541.

Burits, M. and F. Bucar. 2000. Antioxidant activity of Nigella sativa essential oil. Phytother. Res. 14:323-328

Elmer, W.H., D.A. Johnson, and G.I. Mink. 1996. Epidemiology and management of the diseases causal to asparagus decline. Plant Dis. $80: 117-125$

Folin, O. and W. Denis. 1915. A colorimetric method for the determination of phenols (and phenol derivatives) in urine. Biol. Chem. 22: 305-308.

Fridovich, I. 1986. Biological effects of the superoxide radicals. Arch. Biochem. Biophys. 247: $1-11$.

Garmendia, I., J. Aguirreolea, and N. Goicoechea. 2006. Defence-related enzymes in pepper roots during interactions with arbuscular mycorrhizal fungi and/or Verticillium dahliae. Biocont. 51:293-310

Hamel, H., V. Vujanovic, A. Nakano-Hylander, R. Jeannotte, and M. St-Arnaud. 2005. Factors associated with fusarium crown and root rot of asparagus outbreaks in Quebec. Phytopathology 95:867-873.

Hartung, A.C., M.G. Nair, and A.R. Putnam. 1990. Isolation and characterization of phytotoxic compounds from asparagus (Asparagus officinalis L.) roots. J. Chem. Ecol. 16:17071718.

Hichem, H., D. Mounir, and E.A. Naceur. 2009. Defferential responses of two maize (Zea mays L.) varieties to salt stress: Changes on polyphenols composition of foliage and oxidative damages. Ind. Crops Prod. 30:144-151.

Knaflewski, M., P. Golinski, M. Kostecki, A. Waskiewicz, and Z. Weber. 2008. Mycotoxins and mycotoxin-producing fungi occurring in asparagus spears. Acta Hort. 776:183-189.

Lake, R.J., P.G. Falloon, and D.W.M. Cook. 1993. Replant problem and chemical components of asparagus roots. N. Z. J. Crop Hort. Sci. 21: 53-58.

Marschner, H. and B. Dell. 1994. Nutrient uptake in mycorrhizal symbiosis. Plant Soil 159:89102.

Matsubara, Y., N. Hasegawa, and N. Ohba. 2003. Relation between fiber and pactic substance in root tissue and tolerance to fusarium root rot in asparagus plants infected with arbuscular mycorrhizal fungus. J. Jpn. Soc. Hort. Sci. 72 : 275-280.

Miller, H.G., M. Ikawa, and L.C. Peirce. 1991. Caffeic acid identified as an inhibitory compound in asparagus root filtrate. HortScience 26:1525-1527.

Moghaddam, B., M.T. Charles, O. Carisse, and S. Khanizadeh. 2006. Superoxide dismutase responses of strawberry cultivars to infection by Mycosphaerrella fragariae. J. Plant Physiol. 163:147-153.

Nahiyan, A.S.M., L.R. Boyer, P. Jeffries, and Y. Matsubara. 2011. PCR-SSCP analysis of fusarium diversity in asparagus decline in Japan. Eur. J. Plant Pathol. 130:197-203.

Norman, J.R., D. Atkinson, and J.E. Hooker. 1996. Arbuscular mycorrhizal fungal-induced alteration to root architecture in strawberry and induced resistance to the root pathogen Phytophthora fragariae. Plant Soil 185:191198.

Ozgonen, H. and A. Erkilic. 2007. Growth enhancement and Phytophthora blight (Phytophthora capsici Leonian) control by arbuscular mycorrhizal fungal inoculation in pepper. Crop Prot. 26:1682-1688.
Phillips, J.M. and D.S. Hayman. 1970. Improved procedures for clearing roots and staining parasitic and vesicular-arbuscular mycorrhizal fungi for rapid assessment of infection. Trans. Br. Mycol. Soc. 55:158-163.

Pontaroli, A.C. and E.L. Camadro. 2001. Increasing resistance to fusarium crown and root rot in asparagus by gametophyte selection. Euphytica 122:343-350.

Pozo, M.J., C. Cordier, E.D. Gaudot, J.M. Barea, and C.A. Aguilar. 2002. Localized versus systemic effect of arbuscular mycorrhizal fungi on defence responses to Phytophthora infection in tomato plants. J. Expt. Bot. 53: 525-534.

Reid, T.C., M.K. Hausbeck, and K. Kizilkaya. 2002. Use of fungicides and biological controls in the suppression of fusarium crown and root rot of asparagus under green house and growth chamber conditions. Plant Dis. 86: 493-498.

Richter, J., H. Baltruschat, K. Kabrodt, and I. Schellenberg. 2011. Impact of arbuscular mycorrhiza on the St. John's wort (Hypericum perforatum) wilt disease induced Colletotricuhum cf. gloeosporioides. J. Plant Dis. Prot. 118:109-118.

Roe, J.H., B.M. Mary, M.J. Oesterking, and M.D. Charlotle. 1948. The determination of diketoL-gluconic acid, dehydro-L-ascorbic acid, and $\mathrm{L}$-ascorbic acid in the same tissues by 2,4 dinitrophenyl hydrazine method. Biol. Chem. 174:201-208.

Sahoo, M.R., A.M. Dasgupta, A. Paresh, C. Kole, A. Jayant, S. Bhat, and A. Mukherjee. 2007. Antioxidative enzymes and isozymes analysis of taro genotypes and their implications in Phytophthora blight disease resistance. Mycopathologia 163:241-248.

Salzer, P., H. Corbiere, and T. Boller. 1999. Hydrogen peroxidase accumulation in Medicago truncatula roots colonized by the arbuscular mycorrhiza-forming fungus Glomus intraradices. Planta 208:319-325.

Sutton, J.C. 1973. Development of vesiculararbuscular mycorrhizae in crop plants. Can. J. Bot. 51:2487-2493.

Vanitha, S.C., S.R. Niranjana, and S. Umesha. 2009. Role of phenylalanine ammonia lyase and polyphenol oxidase in host resistance to bacterial wilt of tomato. J. Phytopathol. 157: 552-557.

Volpin, H., D.A. Phillips, Y. Okon, and Y. Kapulnik. 1995. Suppression of an isoflavonoid phytoalexin 
defense response on mycorrhizal alfalfa roots. Plant Physiol. 108:1449-1454.

Wong, J.Y. and P. Jeffries. 2006. Diversity of pathogenic fusarium populations associated with asparagus roots in decline soils in Spain and the UK. Plant Pathol. 55:331-342.

Wu, Q., Y. Zou, and R. Xia. 2006. Effect of water stress and arbuscular mycorrhizal fungi on reactive oxygen metabolism and antioxidant production by citrus (Citrus tangerine) roots. Eur. J. Soil Biol. 42:166-172.

Yergeau, E., V. Vujanovic, and M. St-Arnaud. 2006. Changes in communities of fusarium and arbuscular mycorrhizal as related to different asparagus cultural factors. Microb. Ecol. 52:104-113.
Yong, C.C. 1984. Autointoxication in root exudates of Asparagus officinalis L. Plant Soil 82: 247-253.

Zhu, X., F. Song, and H. Xu. 2010. Influence of arbuscular mycorrhiza on lipid peroxidation and antioxidative enzyme activity of maize plants under temperature stress. Mycorrhiza 20: 325-332. 